\title{
A combined continuous-wave and pulsed microwave copper chloride discharge
}

\author{
W. McColl, M. Passow, a) and M. L. Brake \\ Department of Nuclear Engineering, University of Michigan, Ann Arbor, Michigan 48109
}

(Received 12 August 1991; accepted for publication 4 November 1991)

\begin{abstract}
Pulsed and continuous-wave microwaves at $2.45 \mathrm{GHz}$ combined in an Asmussen resonant cavity are used to vaporize, dissociate, and excite copper chloride discharges. Steady state microwaves from 50 to $150 \mathrm{~W}$ sustain a microwave discharge which heats and dissociates the copper chloride to a sufficient vapor pressure. A variable frequency $(2.45$ to 2.60 $\mathrm{GHz}$ ) pulsed microwave source with pulse widths ranging from 0.5 to $2 \mathrm{~ms}$, repetition rates of 500 to $5000 \mathrm{~Hz}$ and a peak output power of 4,500 W then excites the copper atomic states. The two microwave signals are superimposed using a hybrid junction before input into the resonant cavity. Microwave frequencies of the pulsed portion of the signal around $2.50 \mathrm{GHz}$ provided maximum absorption by the discharge. This device is being examined as a potential pump source for a copper vapor laser.
\end{abstract}

\section{INTRODUCTION}

Microwave discharges have been shown to be an effective pump source for excimer ${ }^{1-5}$ and $\mathrm{HeNe}$ lasers. ${ }^{6}$ The benefits of microwave discharges are the absence of electrodes, high coupling efficiency of microwave power to the laser medium, the relatively low cost of microwave components, the high repetition rates needed for pulsed laser sources are within the range of microwave sources, and the ability of microwaves to penetrate deeply into a plasma which allows for large diameters and uniform laser beam profiles. $^{7}$

Current copper vapor lasers ${ }^{8.9}$ ( 510.6 and $578.2 \mathrm{~nm}$ ) and copper chloride lasers ${ }^{10-14}$ use repetitively pulsed electrical discharges between two electrodes to excite a copper vapor. In these systems the electrical discharges also vaporize the copper chloride. This paper presents a new method of heating and exciting copper chloride using microwaves, along with a method for combining a pulsed and continuous-wave $(\mathrm{cw})$ microwave signal in a resonant cavity for laser applications.

\section{EXPERIMENT}

Copper chloride/helium discharges are excited in the transverse-magnetic $\left(\mathrm{TM}_{012}\right.$ ) mode of an Asmussen resonant cavity. ${ }^{15-17}$ The brass resonant cavity has an adjustable length of 6 to $16 \mathrm{~cm}$ which allows for various operating modes and also for shifts in the resonant frequency caused by the discharge. An adjustable coupling probe located in the wall of the cavity excites the standing waves inside the cavity and acts as a variable impedance transformer. A wire mesh window opposite of the coupling probe provides visible access to the plasma discharge, and holes in the cavity wall placed along the axis of the cavity provides for the insertion of diagnostic probes.

The cw microwave source is a Micro-Now magnetron oscillator which operates at $2.45 \mathrm{GHz}$ with continuously

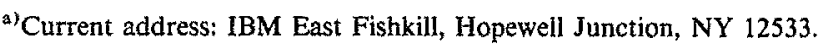

adjustable power ranging from about 50 to $500 \mathrm{~W}$. An (EPSCO) high power pulsed signal source operating from 2.35 to $2.7 \mathrm{GHz}$ provides pulsed microwave signals with pulse widths ranging from 0.5 to $2 \mu \mathrm{s}$, at repetition rates ranging from 500 to 5000 pulses per second for a peak output power of $4500 \mathrm{~W}$. A schematic of the microwave circuit is shown in Fig. 1. Terminated three port circulators protect the two power supplies. A hybrid junction adds the two microwave signals together and dual-directional couplers are used to monitor the power. Coaxial cables with Type $\mathrm{N}$ connections transmit the microwave signals. The $\mathrm{cw}$ power is measured with thermistor sensors monitored by analog power meters. The combined $\mathrm{cw}$ and pulsed signals are measured using crystal diode detectors. A typical power trace is shown in Fig. 2. The total power absorbed by the discharge is taken to be the difference between the measured incident and reflected power. Note that one major disadvantage of this system is that the hybrid junction splits each signal in half. Thus $50 \%$ of both signals is currently disregarded. However, an advantage to this system is that the power levels, pulse width, pulse repetition, and pulse frequency are fully adjustable to suit the plasma conditions.

Typical absorbed powers and operating parameters are shown in Table I. The electron densities, collisional frequencies, and cavity electric and magnetic fields are estimated using an electromagnetic model developed earlier. ${ }^{17,18}$ Figure 3 shows the electric field configuration in the cavity for the $\mathbf{T M}_{012}$ mode as calculated from the model, along with the typical plasma position.

The plasma is contained within a $25 \mathrm{~mm}$ inside diameter (i.d.) $[28 \mathrm{~mm}$ outside diameter (o.d.)] quartz tube located along the axis of the resonant cavity. Helium is used as the buffer gas. (QF) 25 fittings are attached to each end of the tube. A "tee" made up of three QF fittings is attached to each end of the tube, as shown in Fig. 4. Gas is supplied to one tee and a vacuum system is attached to the other tee. Two Lucite windows, or Brewster's windows, attached to the tee ends allows for viewing the plasma along the axis of the tube. 


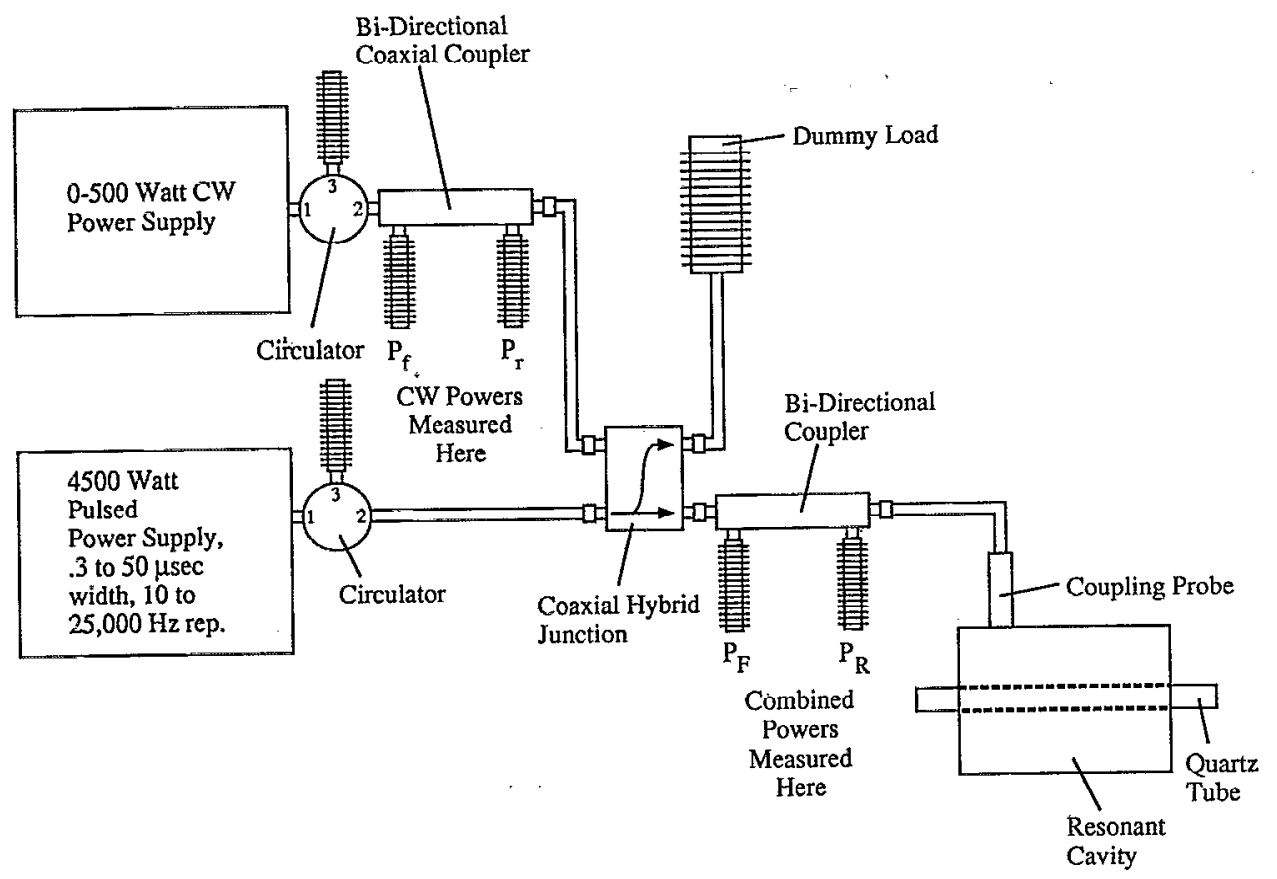

FIG. 1. The microwave circuit used to superimpose the continuous wave and pulsed microwave power sources. One disadvantage to this system is that half of the total power is disregarded.

Cupric chloride $\left(\mathrm{CuCl}_{2}\right)$ with a melting temperature of $695 \mathrm{~K}$ is used as the copper source. Two methods are used to obtain a copper chloride vapor. The first uses a resistive tape to heat a reservoir of copper chloride crystals upstream of the resonant cavity. This method is difficult to implement because the copper vapor recombines on the walls of the discharge tube before reaching the discharge region.

In the second method, a small amount of copper chloride powder is placed directly inside the quartz tube in the center of the discharge region of the cavity. A cw microwave signal at $2.45 \mathrm{GHz}$ and about $70 \mathrm{~W}$ absorbed power heats the helium buffer gas to temperatures of $800 \mathrm{~K}$ and higher. The helium collisionally transfers the thermal energy to the copper chloride which is then vaporized and

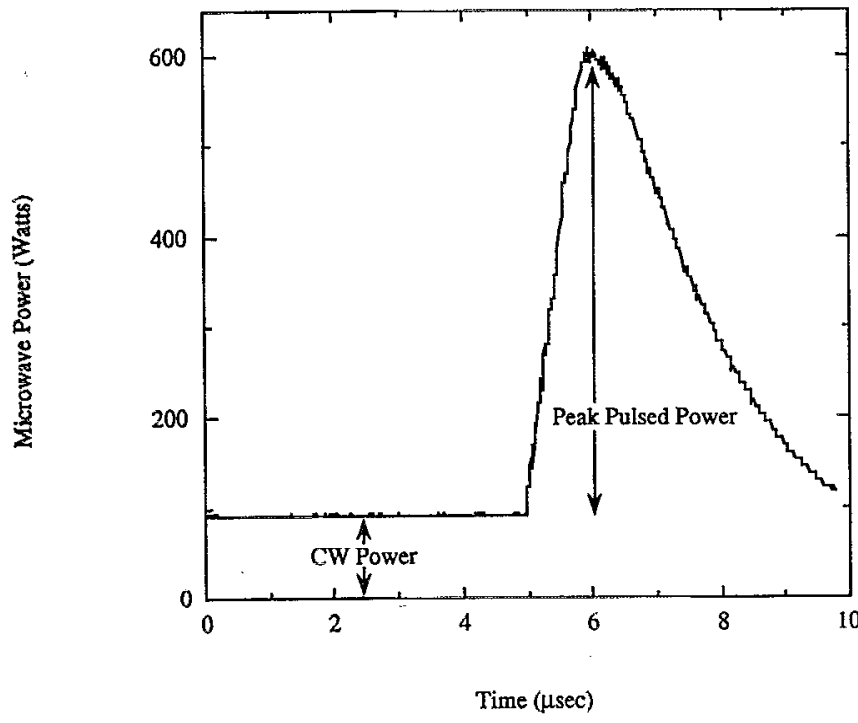

FIG. 2. A typical power trace of the combined microwave signal showing the $\mathrm{cw}$ and pulsed microwaves. dissociated. Temperature gradients across the quartz tube wall often cause the copper chloride discharge to become unstable. To overcome this, glass wool (quartz) was wrapped around the tube to provide insulation. The addition of the glass wool greatly increases the stability of the discharge. A disadvantage to this is that the glass wool reduces the quality or $Q$ factor of the cavity.

The copper vapor density is approximated by an estimate of the temperature of the discharge and the vapor pressure of the copper chloride, which is a function of temperature as given in Ref. 19. It is assumed that the $\mathrm{cw}$ portion of the microwave signal provides the majority of the heating. A simple energy conservation equation can be written as: ${ }^{20}$

$$
\nabla \cdot(\kappa \nabla T)+P_{d}=0,
$$

where $T$ is the discharge neutral gas temperature, $\kappa$ is the thermal conductivity and $P_{d}$ is the dissipated power. We also assume that helium constitutes most of the discharge, or that the copper chloride content is negligible compared to the helium content. The thermal conductivity for helium is found in Ref. 21 and is fit to $\kappa=a T^{1 / 2}+b$, a typical

TABLE I. Typical operating parameters of the copper chloride microwave discharges.

\begin{tabular}{lr}
\hline \hline Resonant frequency & $2.45 \mathrm{GHz}$ \\
cw power & 50 to $150 \mathrm{~W}$ \\
Peak pulsed power & 80 to $510 \mathrm{~W}$ \\
Pulse width & 0.5 to $2 \mu \mathrm{s}$ \\
Pulse frequency & 2.45 to $2.6 \mathrm{GHz}$ \\
Pulse repetition & 500 to $5000 \mathrm{~Hz}$ \\
Buffer gas & Helium \\
Buffer pressure & 2 to $100 \mathrm{Torr}$ \\
Neutral gas temperature & 750 to $820 \mathrm{~K}$ \\
Electron density & $10^{11}$ to $10^{12} \mathrm{~cm}^{-3}$ \\
Collision frequency & $10^{10}$ to $10^{12} \mathrm{~s}^{-1}$ \\
\hline
\end{tabular}




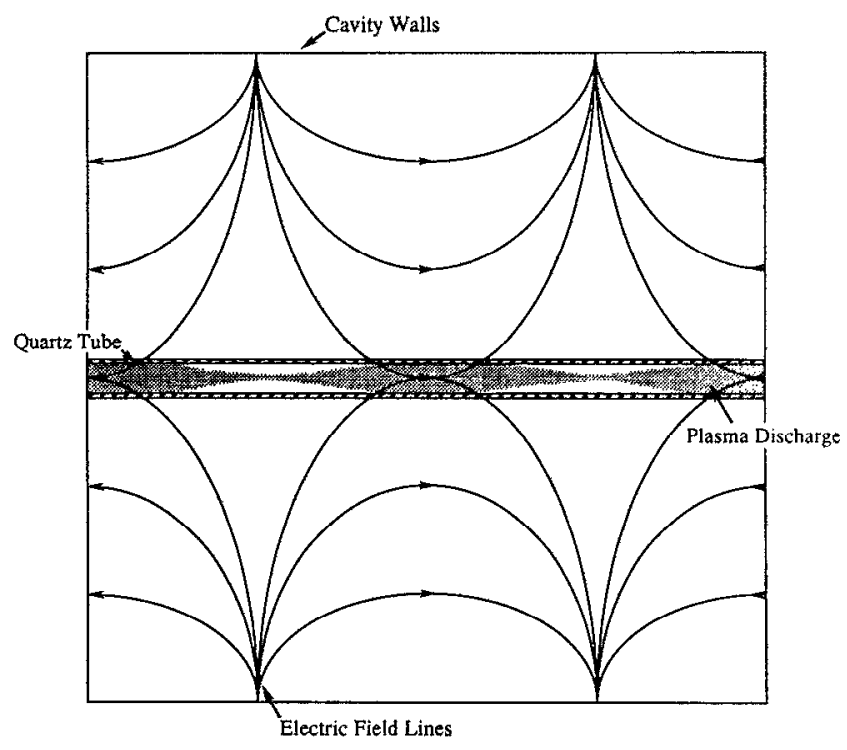

FIG. 3. The electric field lines of a $\mathrm{TM}_{\mathrm{Ol} 2}$ standing wave. The shaded area shows the position of the plasma discharge.

form for atomic gases. ${ }^{22}$ Assuming that $P_{d}(r)$ varies as the ratio of the plasma volume at a radius $r$ to the total plasma volume, i.e., $P_{d}(r)=\left(r^{2} / a^{2}\right) P_{p}$ where $a$ is the radius of the containment tube and $P_{p}$ is the total power absorbed by the plasma, Eq. (1) can be solved for the temperature as a position of radius. An average temperature is defined as the average of the temperature at the wall and at the center of the quartz tube. The wall temperature is assumed to be at most $695 \mathrm{~K}$, the melting point of copper chloride. The estimated gas temperatures were around $780 \mathrm{~K}$ for all pressures (2 to 100 Torr) and powers ( 50 to $100 \mathrm{~W}$ ) studied. This temperature corresponds to a copper vapor pressure of about 1.7 Torr. $^{19}$

The major diagnostic of the copper chloride/helium plasma is optical emission spectroscopy. Light is collected from the end window (along the axis) of the discharge and is imaged onto the entrance slit of a one meter spectrograph coupled to a 1024 channel intensified diode array. The detector is gated by a pulse driver which is triggered by a pulse generator, which also triggers the pulsed microwave supply. By introducing a delay between the power supply trigger and the detector gate pulse, time resolved spectra could be recorded. The width of the detector gate was usually $100 \mathrm{~ns}$.

\section{RESULTS}

The optical emission of the copper chloride/helium plasmas was monitored for a variety of conditions. The laser states (510.6 and $578.2 \mathrm{~nm}$ ) were monitored along with nonlasing states. Figure 5(b) shows a spectra obtained during the $\mathrm{cw}$ portion of the microwave signal. As shown in Fig. 5(a), the lasing transition at $510.6 \mathrm{~nm}$ was greatly enhanced during the pulsed and $\mathrm{cw}$ portion of the microwave signal. Figures 6 and 7 show similar results for the 324.8 and $327.4 \mathrm{~nm}$ pumping transitions and the 578.2 $\mathrm{nm}$ lasing transition, respectively. Obviously, such enhancement of the emissions shows great promise for laser operation. If high enough power densities are obtained, repetitively pulsed laser action should be possible.

The intensity of the copper $510.6 \mathrm{~nm}$ line and the corresponding microwave power is shown in Fig. 8. Note that the peak in this emission occurs during the peak of the microwave power. Similar results were observed for the copper $324.8,327.4$, and $578.2 \mathrm{~nm}$ lines, as well as the helium emission lines.

Adjustment of the frequency of the pulsed microwave signal was necessary to achieve maximum power absorption by the plasma. The value of the pulsed microwave frequency was usually around $2.50 \mathrm{GHz}$, but always greater than $2.45 \mathrm{GHz}$. This upshift in the resonant frequency agrecs with what is expected from theory, as shown by Buchsbaum et al., ${ }^{23}$ who gives the frequency shift as:

$$
\frac{\Delta \omega}{\omega_{0}} \approx \frac{1}{2} \frac{\int\left[\left(\eta /\left(1+\gamma^{2}\right)\right] E_{0}^{2} d V\right.}{\int E_{0}^{2} d V}
$$

where $E_{0}$ is the electric field of an unloaded cavity, $\omega_{0}$ is the resonant frequency of the cavity, $\eta=n_{e} e^{2} / m_{e} \epsilon_{0} \omega_{0}^{2}, n_{e}$ is the electron density, and $\gamma$ is the collisional frequency normalized to the resonant frequency i.e., $v / \omega_{0}$. Persson ${ }^{24}$ showed that this technique could be used up to plasma densities of $10^{12} 1 / \mathrm{cm}^{3}$ if the electric fields are perpendicular to any density gradients. As an approximation, we assume that the fields of the $\mathbf{T M}_{012}$ mode satisfy this requirement. This is a justified because the gradients are in the radial direction and the radius of the plasma is small compared to the radius of the cavity. It is also assumed that the plasma consists entirely of the helium buffer, so that the collision frequency can be calculated from ${ }^{25}$

$$
\left(v_{c}\right)_{H e}=2.37 \times 10^{9} p_{0}
$$

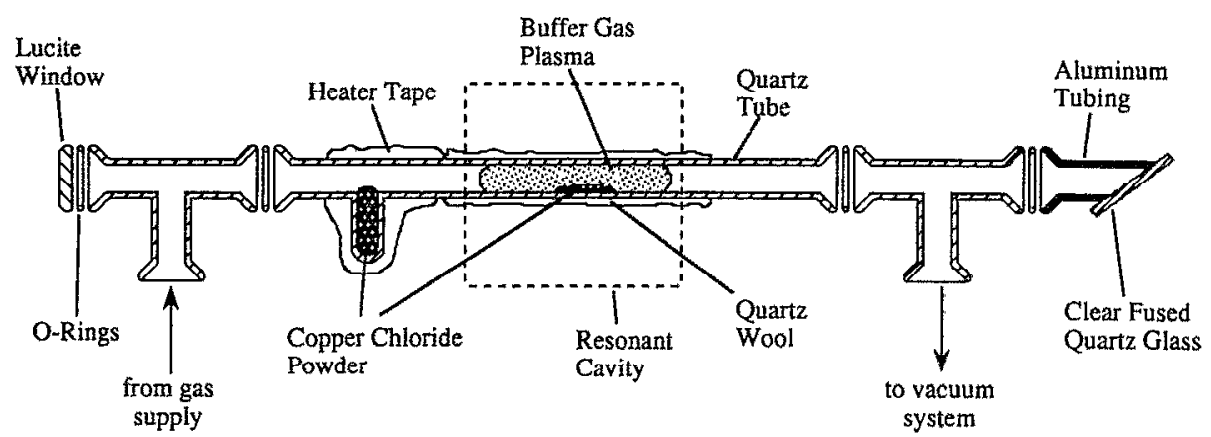

FIG. 4. The quartz tube used to house the plasma discharge. Glass wool was used to insulate the tube to provide high temperatures needed to melt copper chloride. 


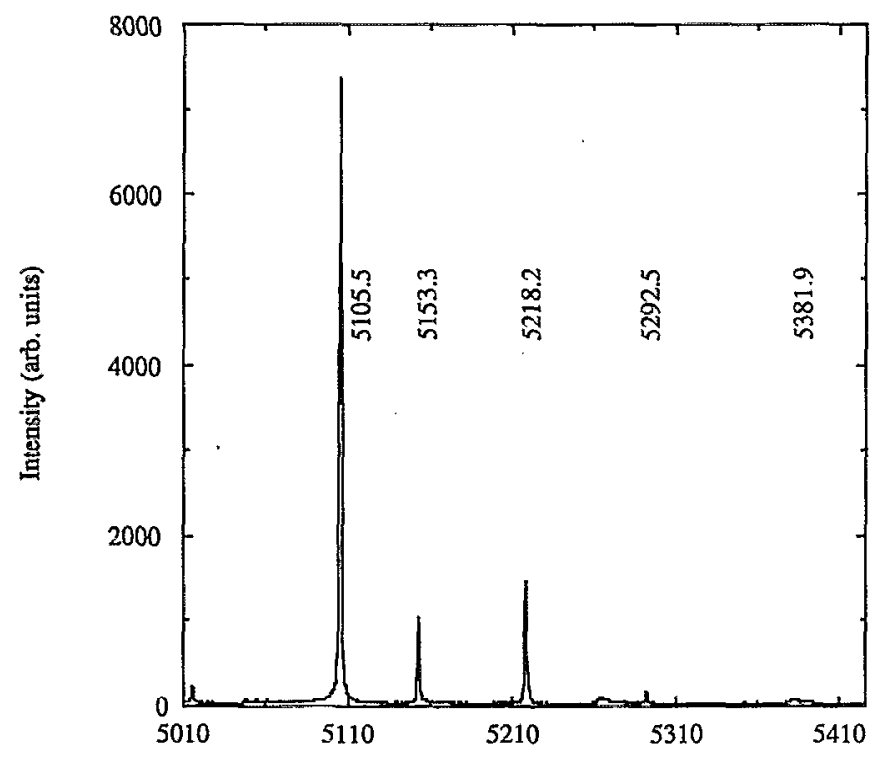

(a)

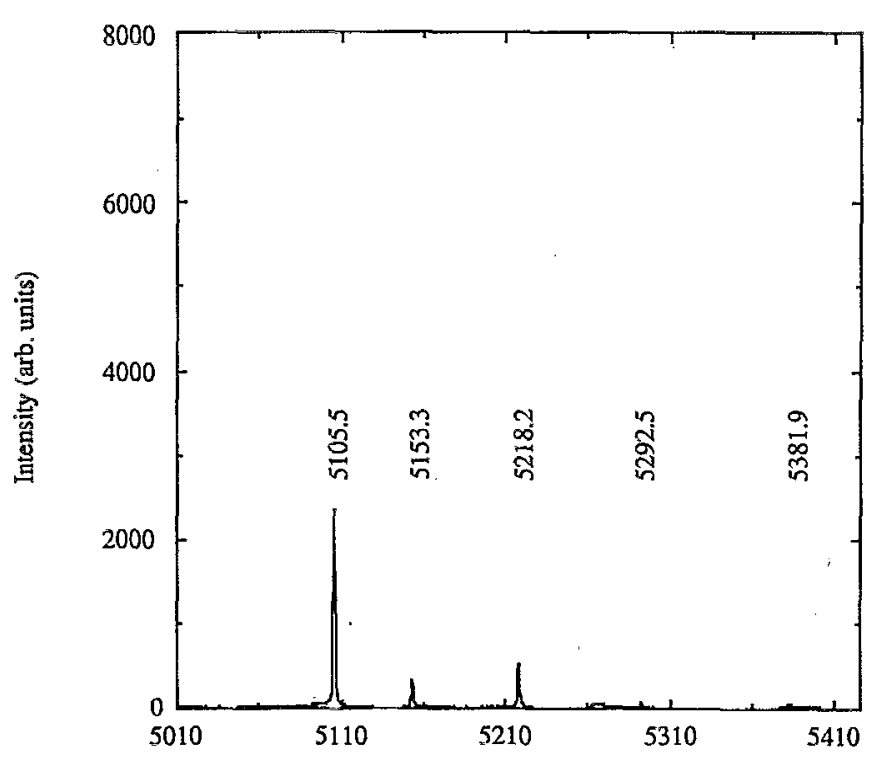

(b)

Wavelength $(\AA)$

FIG. 5. (a) Emission spectra ( 501.0 to $541.0 \mathrm{~nm}$ ) during the microwave pulse with an absorbed cw power of $101 \mathrm{~W}$, an absorbed pulsed power of $90 \mathrm{~W}$, a pulse width of $1 \mu \mathrm{s}$, at a pressure of 2 Torr, and a repetition rate of $500 \mathrm{~Hz}$. (b). Emission spectra ( 501.0 to $541.0 \mathrm{~nm}$ ) during the $\mathrm{cw}$ portion of the microwave signal for the conditions of Fig. 5(b).

$$
p_{0}=p_{m} \frac{273 K}{T_{g}}
$$

where $p_{0}$ is the reduced pressure, $p_{m}$ is the measured buffer gas pressure, and $T_{g}$ is the gas temperature estimated using Eq. (1). Plugging in the electric ficlds for the $\mathrm{TM}_{012}$ mode allows for the calculation of the electron density. Results of this technique predict electron densities of around

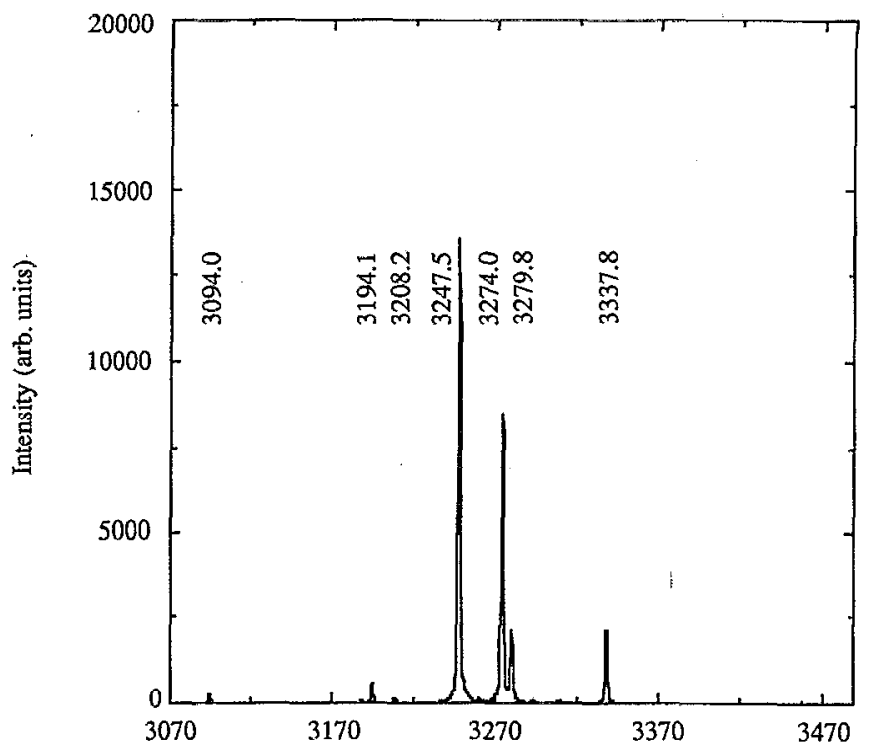

(a)

Wavelength $(\AA)$

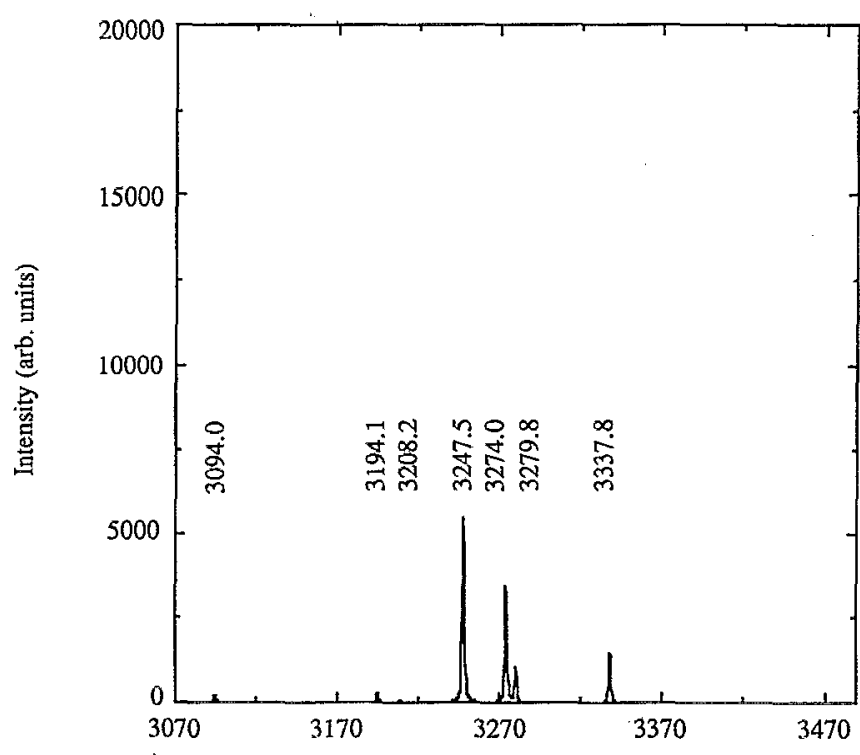

(b)

Wavelength $(\AA)$

FIG. 6. (a) Emisssion spectra ( 307.0 to $347.0 \mathrm{~nm}$ ) during the microwave pulse with an absorbed cw power of $101 \mathrm{~W}$, an absorbed pulsed power of $250 \mathrm{~W}$, a pulse width of $1 \mu \mathrm{s}$, at a pressure of 2 Torr, and a repetition rate of $500 \mathrm{~Hz}$. (b) Emission spectra ( 307.0 to $347.0 \mathrm{~nm}$ ) during the $\mathrm{cw}$ portion of the microwave signal for the conditions of Fig. $6(\mathrm{~b})$.

$2 \times 10^{11} 1 / \mathrm{cm}^{3}$, which agrees with the results of the electromagnetic model mentioned earlier.

\section{DISCUSSION}

Results show that the mixing of cw and pulsed microwaves at $2.45 \mathrm{GIIz}$ is easily accomplished, although power losses encountered are high (at least 50\%). The mixing of the two signals is accomplished using a hybrid junction. 


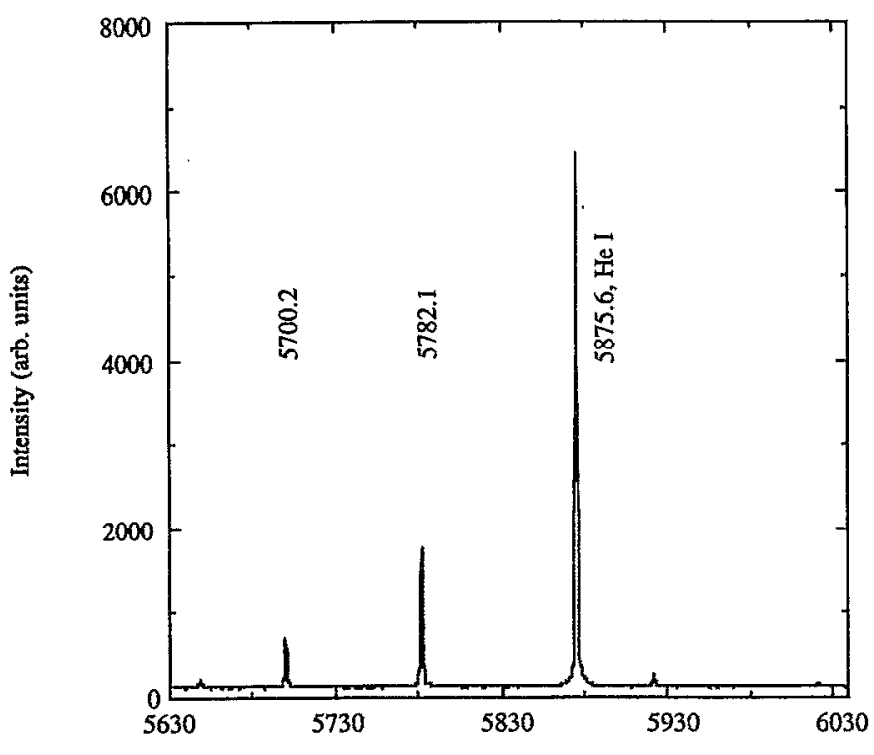

(a)

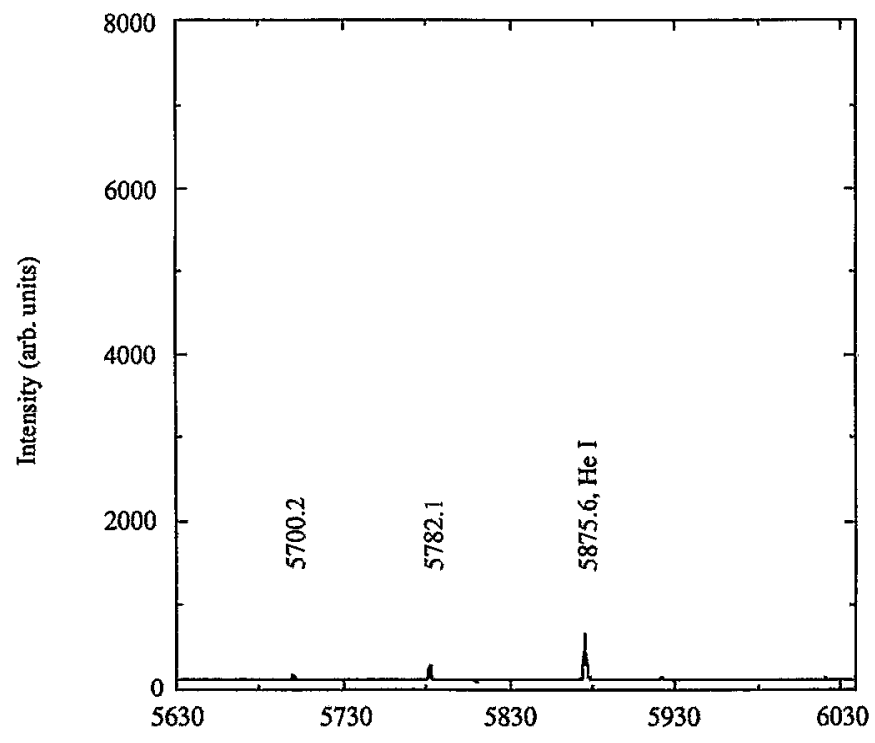

(b)

Wavelength $(\AA)$

FIG. 7. (a) Emission spectra ( 563.0 to $603.0 \mathrm{~nm}$ ) during the microwave pulse with an absorbed $\mathrm{cw}$ power of $93 \mathrm{~W}$, an absorbed pulsed power of $390 \mathrm{~W}$, a pulse width of $1.5 \mu \mathrm{s}$, at a pressure of 2 Torr, and a repetition rate of $1,000 \mathrm{~Hz}$. (b) Emission spectra ( 563.0 to $603.0 \mathrm{~nm}$ ) during the $\mathrm{cw}$ portion of the microwave signal for the conditions of Fig. $7(\mathrm{~b})$.

The combined signal is then used to initiate a plasma discharge in an Asmussen resonant cavity.

Optical spectroscopy is used as a diagnostic to observe the effects of the pulsed microwaves on the plasma. Time resolved spectroscopy indicates that the light emission follows the microwave power, which shows that the pulsed microwaves have a definite effect on the plasma discharge. Enhancement of the intensity of emissions is also shown to be due to the pulsed portion of the microwave signal.

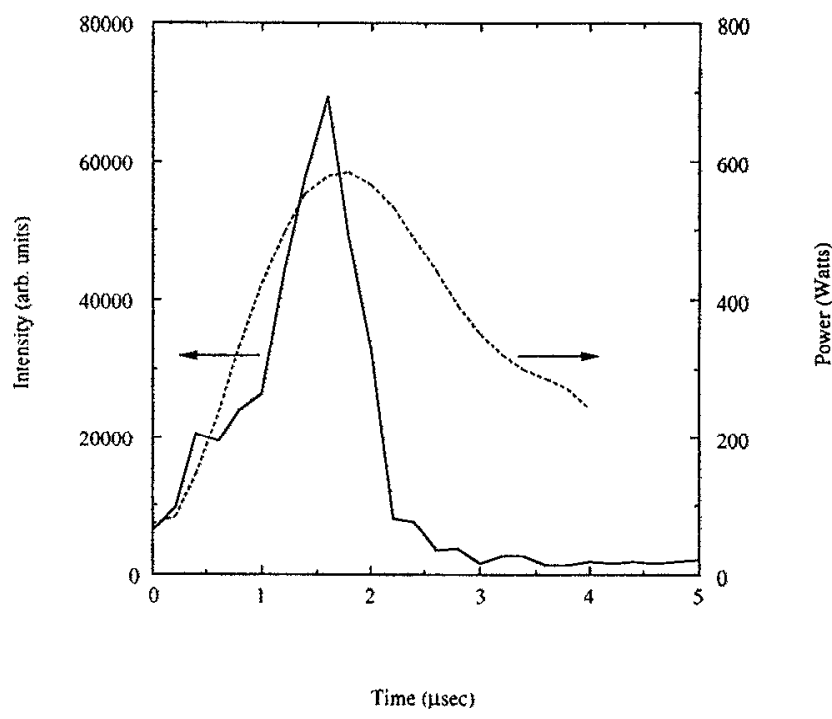

FIG. 8. A time resolved spectra showing the emission of the copper 510.6 nm transition, along with the microwave power trace. The absorbed power was $74 \mathrm{~W}$ with a peak pulsed power of $510 \mathrm{~W}$, a pulse width of 2 $\mu \mathrm{s}$, a pressure of $2.5 \mathrm{Torr}$, and a repetition rate of $1,000 \mathrm{~Hz}$.

Shifts in the resonant frequency of the cavity are also used as diagnostics of the plasma discharge. The shifts are due to the presence of the plasma and a model is used to determine the electron density of the discharges. Results of the model predict electron densities around $2 \times 10^{11}$ $1 / \mathrm{cm}^{3}$.

This concept is presently being applied to a copper chloride discharge in hopes of producing a microwave pumped repetitively pulsed copper vapor laser. Results show that the copper chloride is dissociated by the $\mathrm{cw}$ microwaves, and copper transitions of laser states are increased by the pulsed microwaves.

\section{ACKNOWLEDGMENT}

This work was supported by DOE DE-SG07-89ER 12891.

'C. Gordon, B. Feldman, and C. Christensen, Opt. Lett. 13, 114 (1988).

${ }^{2}$ C. Christensen, W. Waynant, and B. Feldman, Appl. Phys. Lett. 46, 321 (1985).

${ }^{3}$ A. Mendelsohn, R. Normandin, S. Harris, and J. Young, Appl. Phys. Lett. 38, 603 (1981).

${ }^{4} \mathrm{~J}$. Young, S. Harris, P. Wisoff, and A. Mendelsohn, Laser Focus 63 (1982).

${ }^{5}$ C. Christenson and R. Waynant, Appl. Phys. Lett. 41, 794 (1982).

${ }^{6}$ C. Moutoulas, M. Moisan, L. Bertrand, J. Hubert, J. Lachambre, and A. Ricard, Appl. Phys. Lett. 46, 323 (1985).

${ }^{7}$ R. G. Bosisio, C. F. Weissfioch, and M. R. Wertheimer, J. Microwave Power 7, 325 (1972).

${ }^{8}$ J. J. Kim and K. Im, IEEE J. Quantum Electron. 26, 818 (1990).

${ }^{9}$ M. J. Kushner and B. E. Warner, J. Appl. Phys. 54, 2970 (1983).

${ }^{10}$ N. M. Nerheim, J. Appl. Phys. 48, 3244 (1977).

"W. C. Kreye, Appl. Opt. 23, 108 (1984).

${ }^{12}$ W. C. Kreye and F. L. Roesler, Appl. Opt. 22, 927 (1983).

${ }^{13}$ C. J. Chen, N. M. Nerheim, and G. R. Russel, Appl. Phys. Lett. 23, 514 (1973).

${ }^{14}$ K. Srigouri and T. A. Prasada Rao, J. Appl. Phys. 65, 1438 (1989),

${ }^{15}$ J. Asmussen, R. Mallavarpu, J. Hamann, and H. Park, Proc. IEEE 62, 109 (1974). 
${ }^{16}$ R. Mallavarpu, J. Asmussen, and M. C. Hawley, IEEE Trans. Plasma Sci. PS 6, 341 (1978).

${ }^{17}$ W. McColl, M. S. thesis, University of Michigan, 1990.

${ }^{18}$ M. L. Passow, M. L. Brake, P. Lopez, W. McColl, and T. Repetti, IEEE Trans. Plasma Sci. 19, 219 (1991).

${ }^{19}$ R. H Perry and C. H. Chilton, Chemical Engineers' Handbook, 5th ed. (McGraw-Hill, New York, 1973).

${ }^{20}$ J. G. Eden and B. E. Cherrington, J. Appl. Phys. 44, 4920 (1973).
${ }^{21}$ American Institute of Physics, 2nd ed., edited by D. E. Gray (McGrawHill, New York, 1963).

${ }^{22}$ A. B. Cambel, Plasma Physics and Magnetofluidmechanics (McGrawHill, New York, 1963).

${ }^{23}$ S. J. Buchsbaum and S. C. Brown, Phys. Rev. 106, 196 (1957).

${ }^{24}$ K. B. Persson, Phys. Rev. 106, 191 (1957).

${ }^{25} \mathrm{~S}$. C. Brown, Introduction to Electrical Discharges in Gases (Wiley, New York, 1966). 